\title{
Erratum: Fundamental Physics Implications for Higher-Curvature Theories from Binary Black Hole Signals in the LIGO-Virgo Catalog GWTC-1 [Phys. Rev. Lett. 123, 191101 (2019)]
}

Remya Nair, Scott Perkins, Hector O. Silva๑, and Nicolás Yunes

(Q) (Received 6 April 2020; published 24 April 2020)

DOI: 10.1103/PhysRevLett.124.169904

We found an error in Eq. (3) of [1]. The corrected equation is

$$
\beta_{\mathrm{dCS}}=-\frac{5}{8192} \frac{\zeta_{\mathrm{dCS}}}{\eta^{14 / 5}} \frac{\left(m_{1} s_{2}^{\mathrm{dCS}}-m_{2} s_{1}^{\mathrm{dCS}}\right)^{2}}{m^{2}}+\frac{15075}{114688} \frac{\zeta_{\mathrm{dCS}}}{\eta^{14 / 5}} \frac{1}{m^{2}}\left(m_{2}^{2} \chi_{1}^{2}-\frac{350}{201} m_{1} m_{2} \chi_{1} \chi_{2}+m_{1}^{2} \chi_{2}^{2}\right),
$$

which however did not change our conclusion on dynamical Chern-Simons gravity since the modifications to Fig. 1 [1] are minimal. The corrected left panel of Fig. 1 is as follows.

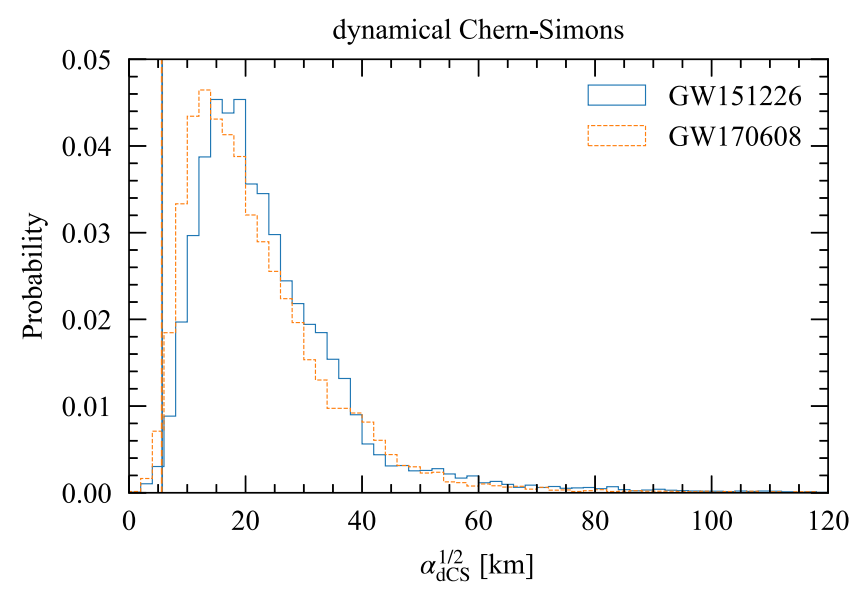

FIG. 1.

We also found a mistake in our calculation for the estimated bound on $\alpha_{\mathrm{dCS}}^{1 / 2}$. The corrected entries from Table I of [1] are

\begin{tabular}{llr}
\hline System & Method & $\alpha_{\mathrm{dCS}}^{1 / 2}[\mathrm{~km}]$ \\
\hline GW151226 & Estimate & 12.6 \\
GW170608 & Estimate & 28.1 \\
\hline
\end{tabular}

All other entries reported in Table I are correct.

[1] R. Nair, S. Perkins, H. O. Silva, and N. Yunes, Phys. Rev. Lett. 123, 191101 (2019). 